\title{
STAND ALONE CONTROLLER FOR LINEAR INTERACTING SYSTEM
}

Stand Alone Algorithm Approach

\author{
P. Rishika Menon ${ }^{1}$, S.Sakthi Priya ${ }^{1}$, G. Brindha ${ }^{2}$ \\ ${ }^{1}$ Department of Electronics and Instrumentation Engineering, St. Joseph's College of Engineering, Chennai- \\ 600119, India \\ ${ }^{2}$ Assistant Professor of Department of Electronics and Instrumentation Engineering, St.Joseph's College of \\ Engineering, Chennai-600119, India \\ 1rish.m95@gmail.com, ${ }^{1}$ sakthi11095@gmail.com,2brindhags30@gmail.com
}

\begin{abstract}
In this paper, we are implementing a PID controller using a VHDL tool.Linear systems have control problems due to linear dynamic behavior, uncertain and time varying parameters. The linear interacting system consists of two cylindrical tanks of equal cross section which can be considered as two first order systems connected in interacting form. The conventional PID controller parameters are calculated using Brambilla and $\mathrm{Bi}$ et al method. And real time implementation of the method in MATLAB and QUATRUS II are done. It is found from the results that the Bi et all method performs better in MATLAB and VHDL based implementation. Systemparameters like peak overshoot, settling time, ISE and IAE are considered for comparison.
\end{abstract}

Keywords - PID controller, Linear system, Interacting system, Brambilla, Bi et al, Vhdl, QUATRUS, Performance parameters

\section{INTRODUCTION}

Conventional proportional-integral-derivative (PID) controller is the most efficient, powerful and economic controller to regulate and control industrial processes [1].The PID controller is able to predict future error and sum the past errors and obtain an effective controller output [3].Therefore, the controller output can be said as the responsiveness to the error value. A linear interacting system is inherently linear in its behavior. When we need to control the level in that system, input variable fluctuation, time delay in the process and many other parameters can pose a problem. Implementing a suitable controller with better tuning method according to the disturbances can lead to effective control of the system. The linear interacting system we have considered is given as follows,

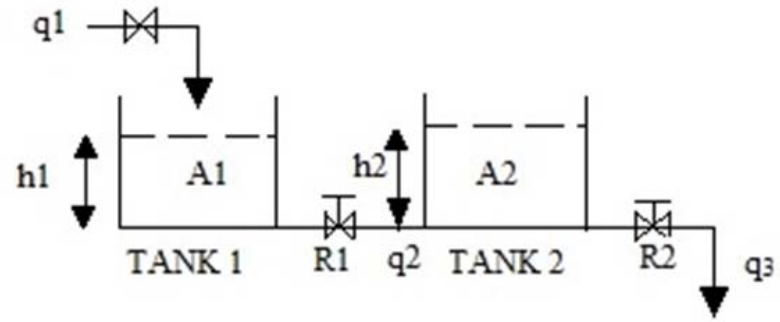

Fig.1. A model of linear interacting system

where,

$\mathrm{q}_{1}=$ inflow to tank 1 in lph

$\mathrm{A}_{1}=$ area of tank 1 in $\mathrm{m}^{2}$

$\mathrm{h}_{1}=$ output variable of tank 1 in $\mathrm{m}$

$\mathrm{R}_{1}=$ resistance of valve head of tank 1

$\mathrm{q}_{2}=$ inflow to tank 2 in lph

$\mathrm{A}_{2}=$ area of tank 2 in $\mathrm{m}^{2}$

$\mathrm{h}_{2}=$ output variable of tank 2 in $\mathrm{m}$

$\mathrm{R}_{2}=$ resistance of valve head of tank 2

$\mathrm{q}_{3}=$ outflow of tank 2 in lph

$\tau_{1}, \tau_{2}=$ time constants of tank $1 \&$ tank 2 respectively

The transfer function of the system is, 


$$
\frac{\mathrm{H}_{2}(\mathrm{~s})}{\mathrm{Q}_{1}(\mathrm{~s})}=\frac{\mathrm{R}_{2}}{\left[\tau_{1} \tau_{2} \mathrm{~s}^{2}+\mathrm{s}\left(\tau_{1}+\tau_{2}+\mathrm{A}_{1} \mathrm{R}_{2}\right)+1\right]}(1)
$$

The system that has been considered for the work is given below,

$$
\frac{\mathrm{H}_{2}(\mathrm{~s})}{\mathrm{Q}_{1}(\mathrm{~s})}=\frac{1.7}{0.918 \mathrm{~s}^{2}+1.93 \mathrm{~s}+1} \mathrm{e}^{-0.5 \mathrm{~s}}(2)
$$

The above mentioned transfer function was taken from the paper [2]. Here, we try to find a better tuning method among the Brambilla and $\mathrm{Bi}$ et al methods to apply to the controller and implement the same in using a VHDL tool. Adopting better hardware area estimation techniques and power reduction techniques yield a much faster and efficient implementation.

\section{PID CONTROLLER}

Tuning a control loop is the conformity of its control parameters to the ideal qualities for the wanted control reaction. A PID controller is the most used controller for the industrial controller for tuning purpose in its actual or slightly varied form.All controllers decide their yield by watching the error between the set point and an estimation of the procedure variable. Occurrence of error can be possibly because of set point changes, outside unsettling influences on process factors and so forth. The controller's main objective is to estimate the error and rectify them automatically. A PID controller principally consists of three terms namely Proportional $\left(\mathrm{K}_{\mathrm{p}}\right)$, Integral $\left(\mathrm{K}_{\mathrm{d}}\right)$ and Derivative term $\left(\mathrm{K}_{\mathrm{d}}\right)$. The sum of these terms is used to calculate the controller output. The parallel form of the controller is represented as,

$$
Y(t)=K_{p} e(t)+K_{i} \int_{0}^{\tau} e(\tau) d t+K_{d} \frac{d e(t)}{d t}(3)
$$

Another form of PID controller is,

$$
\begin{aligned}
& Y(t)=K\left[e(t)+\frac{1}{T_{i}} \int_{0}^{\tau} e(\tau) d t+T_{d} \frac{d e(t)}{d t}+\right](4) \\
& Y(t)=\text { controller output } \\
& e(t)=\text { error value, difference between process variable and reference } \\
& K_{p}=\text { proportional gain } \\
& K_{i}=\text { integral gain } \\
& K_{d}=\text { derivative gain }
\end{aligned}
$$

The controller equation in $S$-domain can be written as,

$$
\begin{aligned}
& Y(t)=K_{p} e(t)+K_{i} \int_{0}^{\tau} e(\tau) d t+K_{d} \frac{d e(t)}{d t}(5) \\
& Y(t)=K_{p} e(t)+K_{i} \int_{0}^{\tau} e(\tau) d t+K_{d} \frac{d e(t)}{d t}(6)
\end{aligned}
$$

We are converting the parameters from one form to another by,

$$
\begin{aligned}
\mathrm{K}_{\mathrm{p}} & =\mathrm{K} \\
\mathrm{K}_{\mathrm{i}} & =\frac{\mathrm{K}}{\mathrm{T}_{\mathrm{i}}} \\
\mathrm{K}_{\mathrm{d}} & =\mathrm{K} * \mathrm{~T}_{\mathrm{d}}
\end{aligned}
$$

The proportional terms acts on the current error value, the integral term accounts for the past errors and the derivative term anticipates the future errors. 


\section{CONTROLLER TUNING}

The tuning parameters of a controller decide the performance of a system. Henceforth determination of appropriate controller and tuning techniques is imperative. Here, we have considered a second order system with a time delay,

Where,

$$
P(s)=\frac{K_{m}}{\left(\tau_{1} s+1\right)\left(\tau_{2} s+1\right)} e^{-t_{d} s}(7)
$$

$$
\begin{aligned}
& \mathrm{K}_{\mathrm{m}}=\text { gain } \\
& \tau_{1}, \tau_{2}=\text { time constants }
\end{aligned}
$$

According to these system parameters, we have to choose apt tuning methods to obtain desired yield. A closed loop control is adopted here, hence, the closed loop control contains the following blocks,

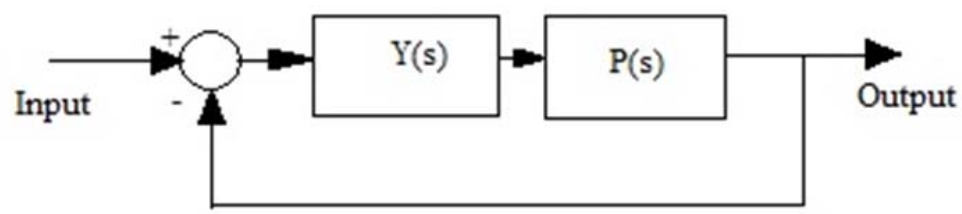

Fig.2. Block diagram of closed loop system

The control loop shown above is designed using MATLAB. It consists of the input block which is a step input, a PID controller, the process plant and the output block which is a scope. The tuning rules may be chosen on following grounds:

- Tuning rules that give a predetermined response

- Tuning rules to minimize specified performance criterion

- Tuning rules based on measured step response

\section{TUNING METHODS}

It is crucial to consider the system parameters and purpose of our tuning method before selection of a tuning method. Henceforth we are considering two tuning methods which are comply with the system parameters such as time constants delay etc, namely, Bi et al and Brambilla method.

\section{A. Bi et all method}

This is first tuning method that is chosen for the controller. This technique of calculating tuning parameters consists of the phrases inclusive of damping consistent, time constants and time delays. Consequently, a higher time area traits can be obtained. This additionally leads to higher ITAE values. The formulation for calculating the controller parameters are given beneath

$$
\begin{aligned}
& \mathrm{K}_{\mathrm{p}}=\frac{1.0128 \varepsilon_{\mathrm{m}} \tau_{1}}{\mathrm{~K}_{\mathrm{m}} \mathrm{t}_{\mathrm{d}}} \\
& \mathrm{T}_{\mathrm{i}}=1.9747 \mathrm{~K}_{\mathrm{m}} \mathrm{t}_{\mathrm{d}}(9) \\
& \mathrm{T}_{\mathrm{d}}=\frac{0.5064 \tau_{1}^{2}}{\mathrm{~K}_{\mathrm{m}} \mathrm{t}_{\mathrm{d}}}(10)
\end{aligned}
$$

\section{B. Brambilla method}

The second method chosen is Brambilla method.

$$
\begin{aligned}
& \mathrm{K}_{\mathrm{p}}=\frac{\tau_{1}+\tau_{2}+0.5 \mathrm{t}_{\mathrm{d}}}{\mathrm{K}_{\mathrm{m}} \mathrm{t}_{\mathrm{d}}(2 \lambda+1)}(11) \\
& \mathrm{T}_{\mathrm{i}}=\tau_{1}+\tau_{2}+0.5 \mathrm{t}_{\mathrm{d}}(12)
\end{aligned}
$$




$$
\mathrm{T}_{\mathrm{d}}=\frac{\tau_{1} \tau_{2}+0.5\left(\tau_{1}+\tau_{2}\right) \mathrm{t}_{\mathrm{d}}}{\tau_{1}+\tau_{2}+0.5 \mathrm{t}_{\mathrm{d}}}(13)
$$

\section{RESULTS AND DISCUSSION}

Proportional-integral-derivative (PID) controllers are the most effective feedback controllers. PID controller is widely used in industry due to their simplicity and easy to tuning. The output of PID controller is a linear aggregate of the proportional, the derivative and the integral of the error value. For controller tuning, the PID parameters are tuned with aid of any traditional methods so as to obtain an appropriate controller output and to gain favoured overall performance.

\section{A. Simulation in Matlab}

Process simulations used for the design, development and analysis and optimization of technical processes such as: chemical plants, chemical processes, environmental systems, power stations, complex manufacturing operations, biological processes and similar technical functions. Thisstudy is to get the optimum PID controller parameters for second order method. Two methods we use are Brambilla and Bi et al methods. Both methods are compared to get the optimum condition for the process model. The Simulation is carried out using MATLAB (version 6.1) for set point tracking and for load rejection.

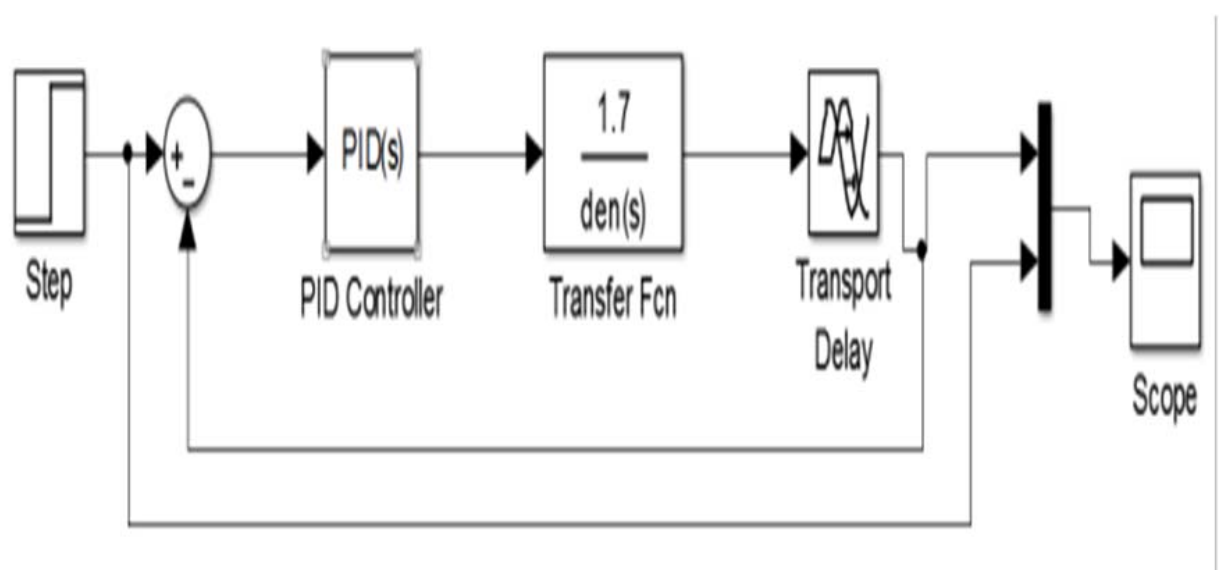

Fig. 3 .Block diagram for simuation in matlab

Performance parameters are obtained and can be tabulated as in TABLE I.

TABLE I

Controller parameters

\begin{tabular}{|l|l|l|l|}
\hline \multicolumn{1}{|c|}{ Tuning method } & \multicolumn{1}{|c|}{$\mathbf{K}_{\mathbf{P}}$} & \multicolumn{1}{|c|}{$\mathbf{K}_{\mathbf{I}}$} & $\mathbf{K}_{\mathbf{p}}$ \\
\hline Bi et al & 1.1474 & 1.6785 & 0.4826 \\
\hline Brambilla & 1.3159 & 2.17 & 0.6442 \\
\hline
\end{tabular}

Response of both the tuning methods is obtained and thereby can be compared from the Fig. 4 . 


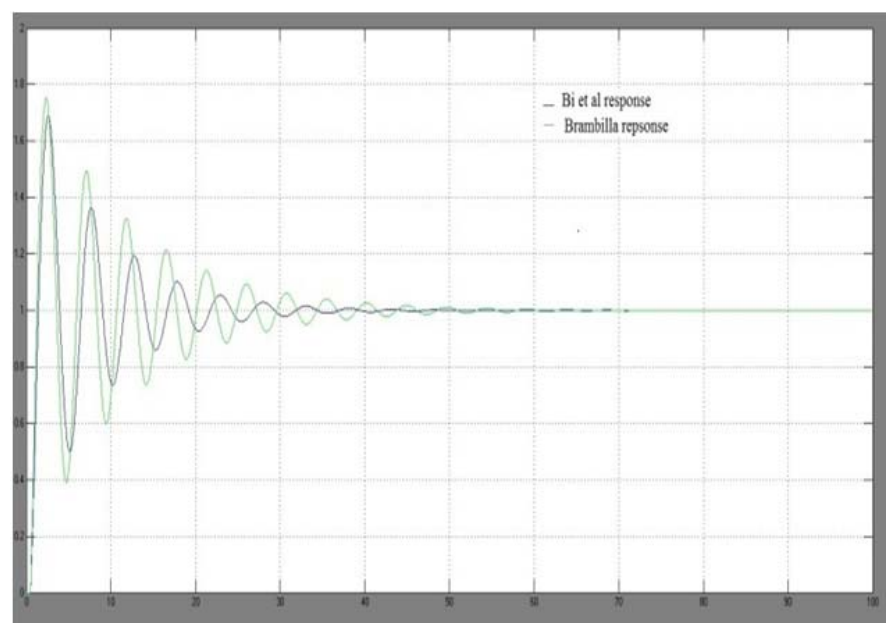

Fig.4. Response of Matlab simulation

\section{B.Comparison based on performance parameters}

Our approach is in development of controller design relation based on a performance index that considers the entire closed loop response. Such indices include

1) Integral of absolute value of error (IAE)

$\mathrm{IAE}=\int_{0}^{\infty}|e(t)| d t$

2) Integral of the square value of the error (ISE)

ISE $=\int_{0}^{\infty} \mathrm{e}^{2} \mathrm{dt}$

These formulas indicate that the integral time depends more on theeffective process time constant and less onthe process dead time.

Simulation for the determination of error detection can be considered and the block diagram for this purpose is provided in fig5.

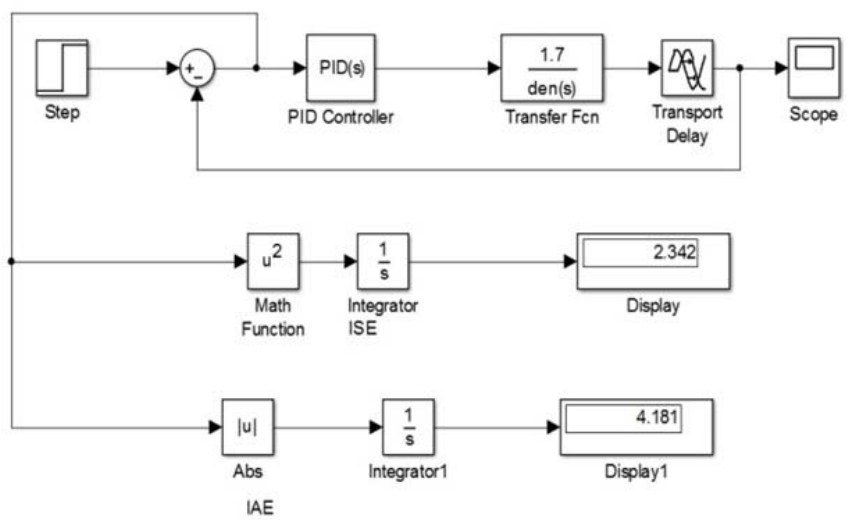

Fig. 5 General Block diagram for error detection 
For both the methods performance analysis has been done and tabulated below,

TABLE II

Comparison of performances indices

\begin{tabular}{|l|c|c|l|l|}
\hline Tuning method & ISE & IAE & Settling time & $\begin{array}{c}\text { Peak } \\
\text { overshoot }\end{array}$ \\
\hline BI ET AL & 1.952 & 3.639 & 44 & 0.69 \\
\hline BRAMBILLA & 2.342 & 4.181 & 60 & 0.75 \\
\hline
\end{tabular}

IAE and ISE values for different tuning methods can be observed and based on the results the best response with minimum error parameters can be obtained by using the Bi et al method.

\section{C.Synthesis using VHDL}

Synthesis is the process of transforming your HDL design in to a gate level net list, given all specified constraints and optimization settings. In this paper, we put forward an implementation of a PID controller using the VHSIC Hardware Description Language on Quartus II software. The control strategy isapplied to a second order system. The Very High Speed Integrated Circuits Description Language was usedas a programming tool. A Proportional-Integral-Derivative Matlab program was also implemented in order to make a performance comparison.

Simulation responses for both the digital and analog outputs for Brambilla method are given below in the Fig. 6\&Fig. 7.

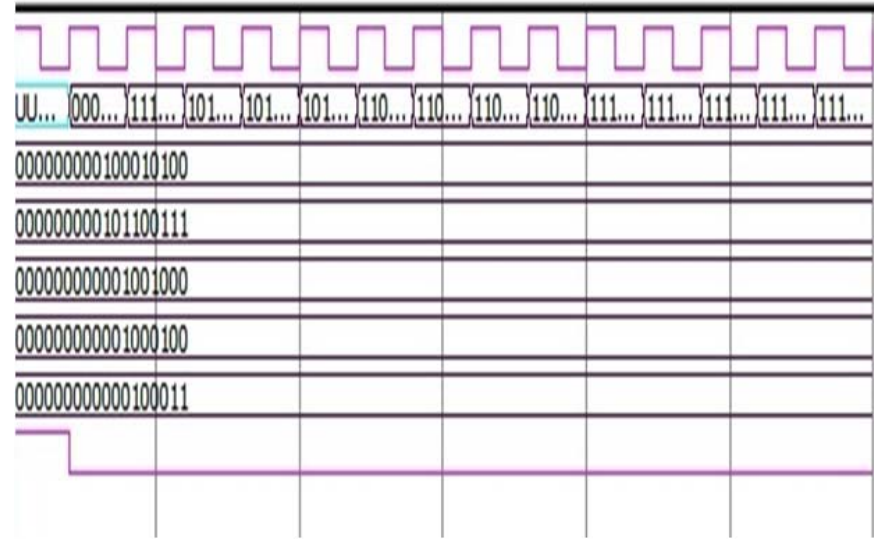

Fig.6. Digital response for Brambilla method

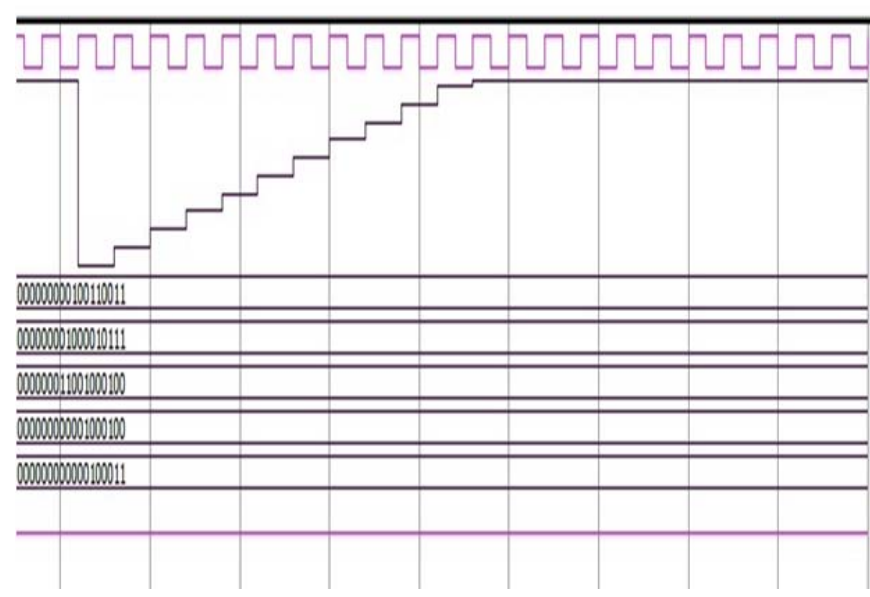

Fig.7. Analog response for Brambilla method 
Simulation responses for both the digital and analog outputs for $\mathrm{Bi}$ et al method are given below in the Fig. $8 \& 9$.

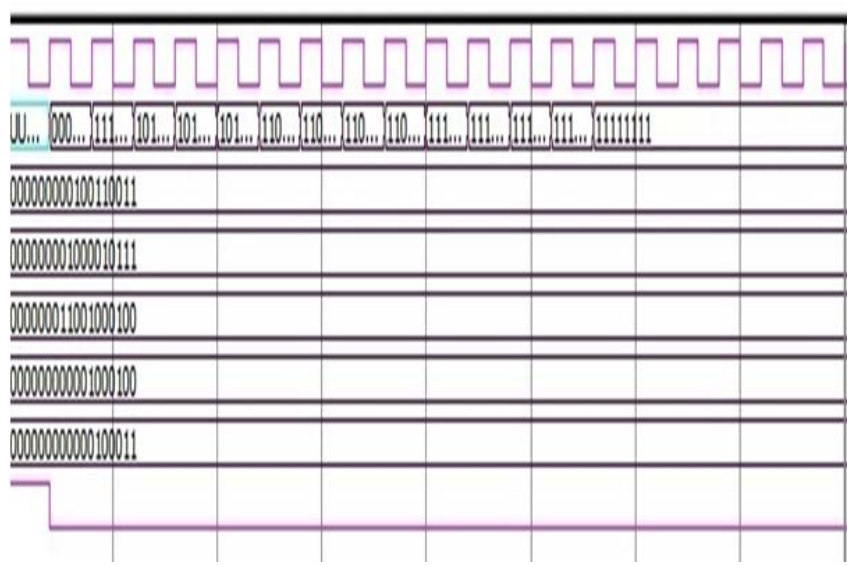

Fig.8. Digital response of Bi et al method

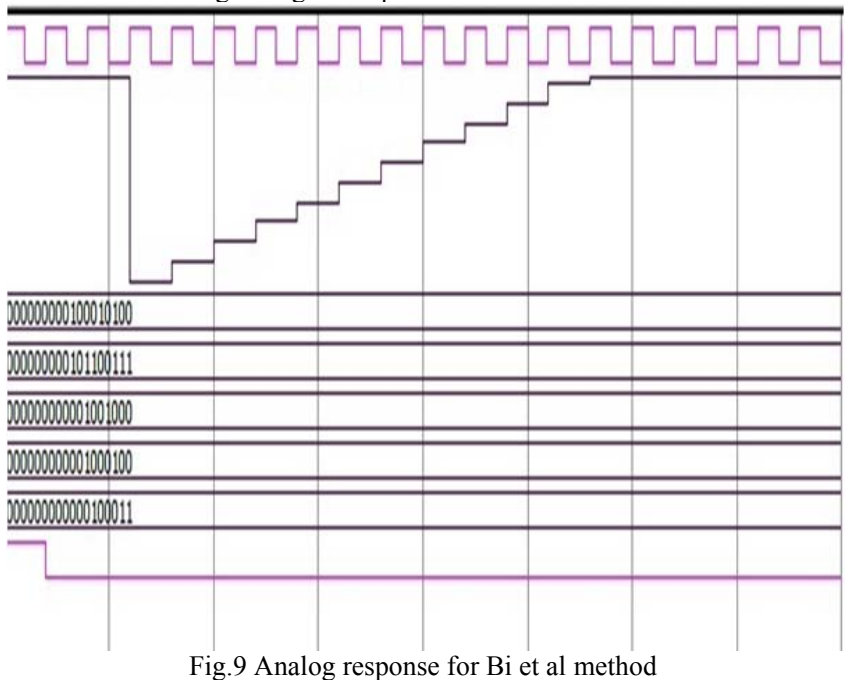

The synthesis using the Quatrus software yields us an RTL schematic diagram which plots out the overall mapping of the input and outputs involved in the controller design. A detailed view of the RTL schematic show all the logic blocks the make up the controller for the specific application.

The basic RTL diagram for controller tuned by $\mathrm{Bi}$ et al method and the detailed view of it are given below in Fig 10 \& Fig 11.

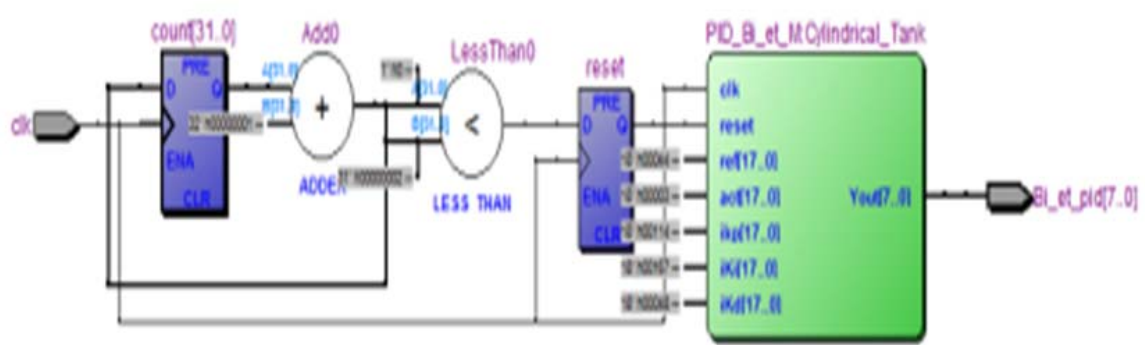

Fig.10. RTL schematic of the controller using Bi et al method 


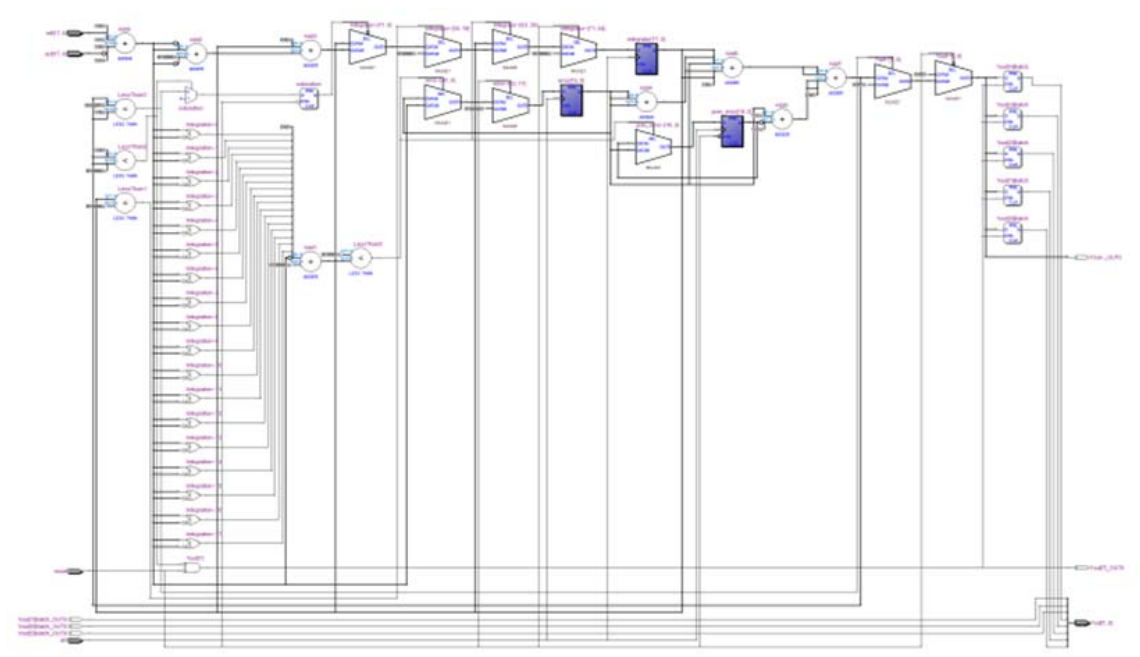

Fig.11. A detailed RTL schematic of the controller using Bi et al method

The basic RTL diagram for controller tuned by Brambilla method and the detailed view of it are given below in Fig 12 \& Fig 13

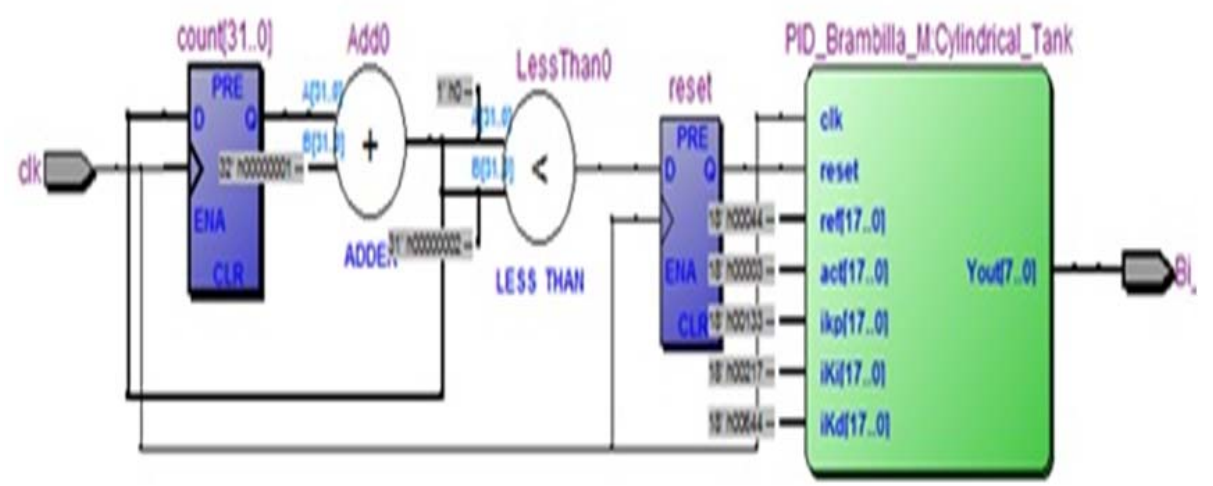

Fig.12. RTL schematic of the controller using Brambilla method

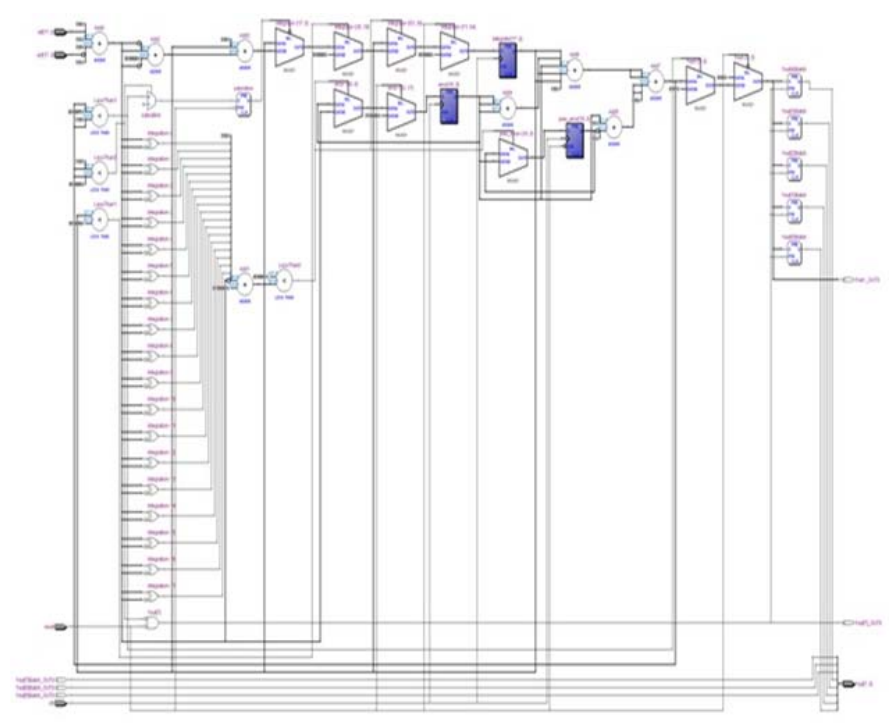

Fig.13. A detailed RTL schematic of the controller using Brambilla method 
Output reaches set point value after 20 iterations in $\mathrm{Bi}$ et al method and 40 iterations in case of Brambilla method. By considering the device utilization summary we can compare the efficiency of both the methods. Device utilization summary is provided in Table III.

TABLE III

Device utilization summary

\begin{tabular}{|l|l|l|}
\hline \multicolumn{1}{|c|}{ Function } & \multicolumn{1}{|c|}{ Brambilla } & \multicolumn{1}{c|}{ Bi et al } \\
\hline Total logic elements used & 109 & 109 \\
\hline Combinational function & 109 & 109 \\
\hline Dedicated logic register & 48 & 48 \\
\hline Number of pins & 9 & 9 \\
\hline Worst-case tco & $19.450 \mathrm{~ns}$ & $15.151 \mathrm{~ns}$ \\
\hline Frequency & $77.89 \mathrm{MHZ}$ & $105.00 \mathrm{MHZ}$ \\
\hline
\end{tabular}

The closed loop test of the PID controller based on VHDL is implemented in Quartus Software and Matlab/Simulink. Synthesis outputs verify to us that it has advantages such as design flexibility, on-line self tuning, highreliability, short period of technical development, high execution speed and robustness which makes it more easily applicable to various industrial control occasions. According to the synthesis response and device utilization summary Bi et al method is more efficient and can be suggested.

\section{CONCLUSION}

This paper conferred about the tuning of PID controller using suitable approaches and thereby compares both the methods and determines the best suited method. In this work, we provide a detailed description about the best suited approach for control of second order interacting system.PID controller tuning is performed using two methods such as Brambilla and Bi et al method.Simulation and synthesis output is obtained. Performances of both the techniques are further validated by time domain and error criteria. Finally from both the responses we obtain that the performance efficiency of Bi et al method is higher compared to that of the Brambilla method. It has a minimum value of error, minimum requirement of space and good performance characteristics with higher stable output.Hence as per our report is considered Bi et al method is suggested for second order interacting system.

\section{REFERENCES}

[1] O’Dwyer, Handbook of PI and PID Controller Tuning Rules, Imperial College Press, London, UK, 3rd edition, 2009.

[2] Bhuvaneswari N S, Praveena R and Divya R, "System Identification and modeling for interacting and non-interacting tank systems using intelligent techniques".

[3] M. A. Johnson and M. H. Moradi, "Some PID control fundamentals," in PID Control: New Identification and Design Methods, chapter 2, pp. 47-107, Springer, London, UK, 2005.

[4] Ms.Rachna Singh, Dr.ArvindRajawat, "A Review of FPGA-based design methodologies for efficient hardware Area estimation", in IOSR Journal of Computer Engineering (IOSR-JCE), 7Volume 13, Issue 4, pp 01-06, e-ISSN: 2278-0661, p- ISSN: 2278-8727, Jul. Aug. 2013.

[5] JulienLamoureux and Wayne Luk, "An Overview of Low-Power Techniques for Field-Programmable Gate Array", in Conference on Adaptive Hardware and Systems.

[6] PrachiRusia ,SandeepBhongade, "Conference on Adaptive Hardware and Systems", in IEEE,2014.

[7] Mohammad Shahrokhi and AlirezaZomorrodi, "Comparison of PID Controller Tuning Methods".

[8] Deep Mukherjee ,Palash Kumar Kundu and ApurbaGhosh, "PID Controller Design for An Interacting Tank Level Process with Time Delay Using MATLAB FOMCON Toolbox" in 2nd International Conference on Control, Instrumentation, Energy \& Communication (CIEC), 2016.

[9] Haider Ali and SulochanaWadhwani "Intelligent PID Controller Tuning for Higher Order Process System", in International Journal of u- and e- Service, Science and Technology, Vol.8, No. 6 (2015), pp.323-330.

[10] Shamsuzzoha M, Lee Moonyong. "Design of advanced PID controller for enhanced disturbance rejection of second-order processes with time delay", AIChE J 2008;54(6):1526-36.

[11] Katarina Paulsson, Michael Hübner, Salih Bayar, Jürgen Becker. "Exploitation of Run-Time Partial Reconfiguration for Dynamic Power Management in Xilinx Spartan III-based Systems".

[12] Vikas Gupta ,KavitaKhare and R. P. Singh, "Efficient Design and Fpga Implementation of Digital Controller Using Xilinx SysGen®" 